\title{
Hapten-enhanced therapeutic effect in advanced stages of lung cancer by ultra-minimum incision personalized intratumoral chemoimmunotherapy therapy
}

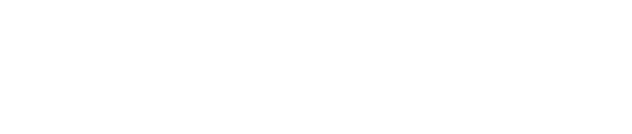

\author{
Baofa Yu ${ }^{1-3}$ \\ Yuanfei Lu' \\ Feng $\mathrm{Gao}^{2}$ \\ Peng Jing ${ }^{2}$ \\ Han Weil \\ Peicheng Zhang ${ }^{2}$ \\ Guoliang Liu' \\ Ning $\mathrm{Ru}^{2}$ \\ Guanghui Cui ${ }^{2}$ \\ Xinhai Xu' \\ Chenglin Sun' \\ Changjiang Guan ${ }^{2}$ \\ Yebing Che ${ }^{1}$ \\ Yingli $\mathrm{Wu}^{2}$ \\ Zhenlu $\mathrm{Ma}^{2}$ \\ Qiang $\mathrm{Fu}^{\prime}$ \\ Jian Liu $^{2}$ \\ Huan-You Wang ${ }^{4}$ \\ Jjinan Baofa Cancer Hospital, Jinan, \\ ${ }^{2}$ TaiMei Baofa Cancer Hospital, \\ Dongping, ${ }^{3}$ Beijing Baofa Cancer \\ Hospital, Beijing, People's Republic \\ of China; ${ }^{4}$ Department of Pathology, \\ University of California, San Diego, \\ CA, USA
}

\begin{abstract}
Aim: The objective of the study reported here was to evaluate the therapeutic effects of hapten-enhanced chemoimmunotherapy in the treatment of advanced lung cancer by ultra-minimum incision personalized intratumoral chemoimmunotherapy (UMIPIC) and to analyze the effect of this immune booster.
\end{abstract}

Materials and methods: A total of 97 patients with advanced lung cancer were treated with UMIPIC or intratumoral chemotherapy (ITCT). UMIPIC was delivered intratumorally in combination with a proprietary therapeutic regimen composed of three components - an oxidant, a cytotoxic drug, and hapten. ITCT applied using the same procedures and regimen, only without hapten. All data from the two groups were reviewed and analyzed. A total of 55 patients were treated with UMIPIC and 42 with ITCT. Patient responses were assessed with computed tomography scan 4-6 weeks after treatment, and all of the patients were followed until their deaths.

Results: Median overall survival was 11.23 months in the UMIPIC (test) group and 5.62 months in the ITCT (control) group $(P<0.01)$. The 6-month and 1-year survival rates of the UMIPIC and ITCT groups were $76.36 \%$ versus $45.23 \%(P<0.01)$ and $45.45 \%$ versus $23.81 \%(P<0.05)$, respectively. Two cycles of UMIPIC treatment $(n=19)$ conferred a significant survival benefit compared with two cycles of ITCT $(n=29)$; significant benefits in survival time were also found with UMIPIC $(n=20)$ compared with ITCT $(n=13)$ when both were utilized without adjuvant treatment.

Conclusion: The hapten-enhanced clinical effect of UMIPIC conferred a superior survival time in patients with advanced lung cancer compared with ITCT. The addition of the hapten in UMIPIC demonstrates a significant advantage in terms of prolonged survival time.

Keywords: hapten-enhanced immunotherapy, intratumoral chemoimmunotherapy, lung cancer, ultra-minimum incision therapy

\section{Highlights}

- Ultra-minimum incision personalized intratumoral chemoimmunotherapy (UMIPIC) can induce more inflammatory responses in local lung cancer tumors and increase survival time.

- It is noninvasive, and potentially effective for high specificity and prolonged survival with acceptable safety.

- Precise treatments can be tailored, especially in advanced/inoperable/drug-resistant lung cancer tumors.
East 50, Wangzhuang Industrial Park, Beijing Baofa Cancer Hospital, Changping, Beijing 102206, People's Republic of China Email bfyuchina@I26.com 


\section{Introduction}

Lung cancer remains the leading cause of cancer-related deaths, accounting for approximately $14 \%$ (228,190 cases) of all cancer cases and 27\% (159,480 deaths) of all cancer deaths in 2013 in the USA. ${ }^{1,2}$ Routine clinical treatments include surgery, radiation therapy, and chemotherapy. The 5 -year survival rate for all stages combined, however, is only $16 \% .{ }^{1}$ Currently, as a first-line treatment with chemotherapy, several agents clinically approved in targeted therapies for lung cancer are in ongoing development such as bevacizumab $\left(\text { Avastin }^{\circledR}\right)^{3}$ and erlotinib (Tarceva ${ }^{\circledR}$ ), as well as the second-generation drugs Gilotrif (BIBW 2992) $)^{4,5}$ and crizotinib (Xalkori $\left.{ }^{\circledR}\right) .{ }^{6}$ However, they still exhibit toxicities and have limitations due to the differences in the molecular and histological profiles of lung cancers. $^{7}$

UMIPIC is a new option for cancer treatment, as it integrates local chemotherapeutic effect with systemic antitumor immunity by intratumoral drug delivery. We have applied UMIPIC in the treatment of advanced lung cancer with a compounded solution including three components: an oxidant, a cytotoxic drug (cytosine arabinoside [Ara-C]), and hapten. ${ }^{8}$ Previous animal studies showed that a clinically approved oxidant can effectively coagulate tumor mass thoroughly by denaturation, which kills more than $90 \%$ of the tumor mass, reduces blood flow, and entraps the injected cytotoxic drugs at a high concentration within the coagulated tumors $(>10 \times$ than conventional chemotherapy) for sustaining drug release. The cytotoxic drug Ara-C can continue to kill tumor cells that were not destroyed by coagulation. At the same time, autologous tumor-associated antigens that are also released from the dead tumor can trigger immune response as a self-vaccination. Meanwhile, hapten binds to the tumor-associated antigens to increase the specificity of these antigens and further boost systematic hormonal and cellular immunity for the suppression and eradication of tumor recurrence and metastasis. In the last decade, we have tried this treatment using combination of drugs with or without hapten in patients with advanced lung cancer. The data have not been published until now, as the combination of two chemotherapeutic drugs and an oxidant with or without hapten in lung cancer treatments was still undergoing clinical research. The data from two groups treated with a single drug have now been collected and analyzed, and the role of hapten in UMIPIC evaluated.

\section{Materials and methods}

\section{Patient selection and data collection}

Patients were informed of details of the study procedure and agreed to participate by signing informed consent and the ethics committee in the author's hospital. The Ethic Committee (EC) approved this study (EC approval letter \#: TMBFZLLYoo1). In total, 97 of 120 patients with advanced-stage lung cancer were enrolled in the two groups for the study (Beijing Baofa Cancer Hospital, Beijing, People's Republic of China). The baseline characteristics of the patients were well balanced between the two groups (Table 1), with no significant differences observed $(P>0.01)$. The complete survival data obtained were analyzed. Test group patients $(\mathrm{n}=55)$ received UMIPIC, with $19 / 55$ of the patients receiving two cycles of treatment with UMIPIC, and 20/55 of the patients receiving UMIPIC without adjuvant treatments. Control group patients $(n=42)$ received intratumoral chemotherapy (ITCT), with 29/42 receiving two cycles of treatment with ITCT, and

Table I Patient baseline characteristics

\begin{tabular}{|c|c|c|c|c|}
\hline \multirow[t]{2}{*}{ Characteristic } & \multicolumn{2}{|c|}{ UMIPIC } & \multicolumn{2}{|c|}{ ITCT } \\
\hline & $\mathbf{N}$ & $\%$ & $\mathbf{N}$ & $\%$ \\
\hline Enrolled patients & 55 & $56.7 \%$ & 42 & $43.3 \%$ \\
\hline \multicolumn{5}{|l|}{ Sex } \\
\hline Male & 43 & $78.1 \%$ & 33 & $78.5 \%$ \\
\hline Female & 12 & $21.8 \%$ & 9 & $21.4 \%$ \\
\hline Median age, years & 59 & & \multicolumn{2}{|l|}{55} \\
\hline Age range, years & \multicolumn{2}{|c|}{$28-76$} & $19-$ & \\
\hline \multicolumn{5}{|l|}{ Histology } \\
\hline Squamous carcinoma & 26 & $47.3 \%$ & 17 & $40.5 \%$ \\
\hline Adenocarcinoma & 15 & $27.2 \%$ & 6 & $14.3 \%$ \\
\hline Large-cell carcinoma & 0 & $0.0 \%$ & I & $2.4 \%$ \\
\hline Cytological diagnosed cancer & 14 & $25.5 \%$ & 18 & $42.8 \%$ \\
\hline \multicolumn{5}{|l|}{ Stage of disease } \\
\hline 1 & 3 & $5.5 \%$ & I & $2.4 \%$ \\
\hline II & 6 & $10.9 \%$ & 5 & $11.9 \%$ \\
\hline III & 30 & $54.5 \%$ & 24 & $57.1 \%$ \\
\hline IV & 16 & $29.1 \%$ & 12 & $28.6 \%$ \\
\hline \multicolumn{5}{|l|}{ Tumor size } \\
\hline$<2 \mathrm{~cm}$ & 4 & $7.3 \%$ & 3 & $7.0 \%$ \\
\hline $2-5 \mathrm{~cm}$ & 20 & $36.3 \%$ & 13 & $30.1 \%$ \\
\hline$>5 \mathrm{~cm}$ & 31 & $56.4 \%$ & 26 & $61.9 \%$ \\
\hline \multicolumn{5}{|l|}{ Adjuvant treatment } \\
\hline Chemotherapy & II & $20.0 \%$ & 11 & $26.2 \%$ \\
\hline Radiotherapy & 18 & $32.7 \%$ & 16 & $38.1 \%$ \\
\hline Prior surgery & I & $1.8 \%$ & 1 & $2.4 \%$ \\
\hline None & 25 & $45.5 \%$ & 14 & $33.3 \%$ \\
\hline \multicolumn{5}{|l|}{ Disease status } \\
\hline Locally advanced & 35 & $63.6 \%$ & 20 & $47.6 \%$ \\
\hline Metastatic disease & 20 & $36.4 \%$ & 22 & $52.4 \%$ \\
\hline
\end{tabular}

Abbreviations: ITCT, intratumoral chemotherapy; UMIPIC, ultra-minimum incision personalized intratumoral chemoimmunotherapy. 
$13 / 42$ of the patients receiving ITCT without adjuvant treatments. Primary lung cancer patients in advanced stages and/or with metastatic cancers (confirmed by imaging and pathologic examination or cytological diagnosis) from November 1999 to September 2006 were analyzed. Data were collected from case report forms filed by physicians from their hospitals. There were three types of data: clinical characteristics data, follow-up time, and response data (Table 1). For each patient, the first follow-up visit was scheduled 1 month after treatment initiation and then scheduled on a monthly basis. The records were updated after each follow-up visit.

\section{ITCT and UMIPIC preparation}

The solution of drugs for UMIPIC was freshly prepared before each intratumoral injection with three clinically approved components: an oxidant, a cytotoxic drug, and hapten. The solution for ITCT contained an oxidant and a cytotoxic drug but no hapten.

\section{Treatment delivery}

All patients had a lung computed tomography (CT) scan as a pretreatment baseline. Routine examination of cardiopulmonary function was also done. Bucinnazine hydrochloride injection $(0.1 \mathrm{~g})$ and hemocoagulase atrox for injection (1 Klobusitzky unit) were injected intramuscularly with the patient lying supine or laterally for accurate location. Prior to UMIPIC, the patients were asked to fast without water for 14 hours prior to this therapy in order to avoid side effects and infections from this therapy.

After routine disinfection, draping, and local anesthesia with $2 \%$ lidocaine, a 25 gauge spinal needle was inserted into the tumor under CT guidance, and the needle tip in the tumor was monitored by CT. The core of the needle was taken out and the inflator was connected and used as a high-pressure syringe (inflation device, $30 \mathrm{~atm} / \mathrm{bar}$; Merit Medical Systems, West Jordan, UT, USA), then the injection of solution was performed.

UMIPIC and ITCT have the same therapeutic procedure, which is minimally invasive and simple like a needle biopsy. The UMIPIC or ITCT was delivered by a spinal needle inserted into the tumor, as just described, and the solution was pressurized (at the level of atmospheric pressure) to obtain the full distribution of the clinically approved regimens in the tumor under CT imaging guidance. A Picker IQ CT unit was used for single-slice scanning and monitoring of the density changes in CT value at a point or area of interest in the lung tumor. Special attention was needed for monitoring the density changes in CT value at the tumor margins to ensure the complete distribution of the drugs. The drugs in the solution are water soluble, which is better than an oil-drug emulsion, which is sticky and hard to distribute in tumors. Under high pressure, the combination of drugs in UMIPIC or ITCT can penetrate the full matrix of the tumor, even into tumor cells, providing sustained drug release for a long time.

The average time the whole procedure took was approximately 30-45 minutes. Patients with severe cough during the treatment were unable to have the procedure completed on them and were excluded from data analysis. The volume of the injection was calculated as the diameter of tumor $\left(D^{t}\right) \times 2$ for $1-5 \mathrm{~cm}$ tumors and $\mathrm{D}^{\mathrm{t}} \times 1.5$ for tumors not smaller than $6 \mathrm{~cm}$; good practice is the key to a successful treatment in all cases according to this calculation in order to deliver enough dosage into tumors (Figure 1).

Having injected the combined solution, the physicians would observe the density values by CT at a point or area of interest of the tumor (indicating drug diffusion in the tumor) and related complications such as hemorrhage around the needle track by CT scan imaging. Second and third cycles of treatment are usually required for better efficacy compared with one cycle of treatment. The patients should be re-examined by CT 4-6 weeks after the last therapy, and some patients in our study were treated with a second cycle of treatment.

\section{Assessment}

The response to treatment was evaluated by the solid tumor effect evaluation criterion of the European Organization for Research and Treatment of Cancer and the Response Evaluation Criteria in Solid Tumors (RECIST) made by National Cancer Institute (USA and Canada) in October 1998. ${ }^{9}$ All case report forms were filed by the treating physicians in the study hospitals.

\section{Statistical analysis}

Statistical analysis was done by Binzhou Medical College (Binzhou, People's Republic of China). The primary objective was to evaluate the overall survival (OS), which was defined as the duration from the first treatment to patient death and was estimated using Kaplan-Meier analysis. The secondary objective was response rate at 4-6 weeks, defined as the proportion of patients with complete response (CR), partial response (PR), or stable disease (SD) after the treatment, using the RECIST (v 1.0). Comparison of effective rate was calculated with the chi-square test. Statistical analysis was conducted with SPSS 


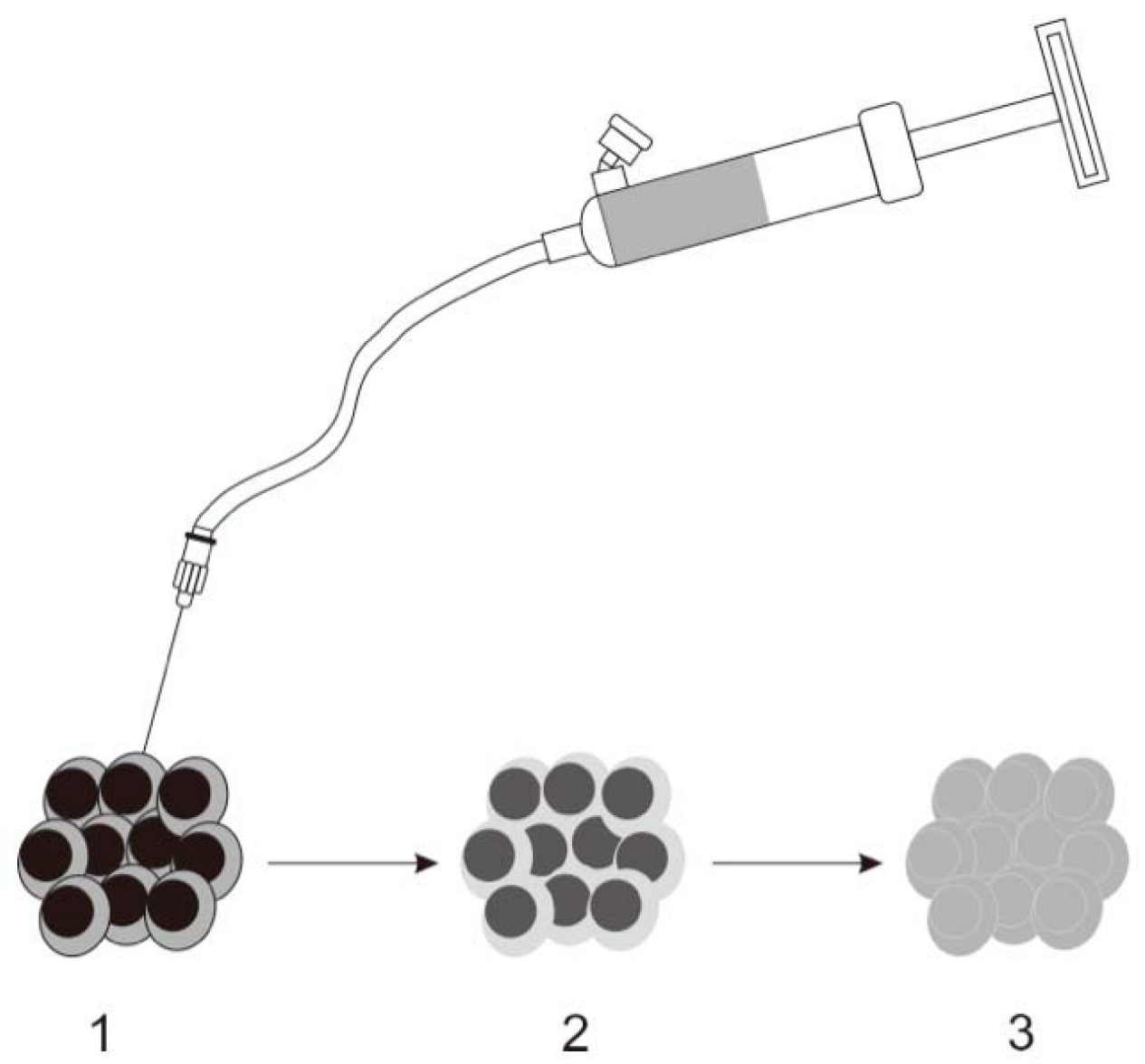

Figure I The ultra-minimum incision personalized intratumoral chemoimmunotherapy procedure.

Notes: I) Guided by computed tomography, the needle is inserted into the tumor, connected to the inflator, and introduced intratumorally with the optimal route and angle; 2) the regimen is slowly delivered into the tumor; 3) with high pressure supplied by the inflator, the solution penetrates the extracellular matrix of the tumor and facilitates diffusion.

statistical software (v 17.0; IBM Corp, Armonk, NY, USA); $P$-values of $<0.05$ were considered of statistical significance.

\section{Results}

\section{Patient characteristics}

At the end of follow-up, 97 of the 120 patients enrolled had completed the study and had their survival data analyzed. Of the 97, 55 patients received UMIPIC and 42 patients received ITCT. In the UMIPIC group, 43 cases were male and 12 were female, and members were aged between 28 and 76 years old, with a median age of $59 \pm 11$ years; $83.6 \%$ were diagnosed as having stage III and IV cancer according to tumor-node metastasis (TNM) classification. Histopathology results indicated that $26(47.3 \%)$ of the patients had squamous cell carcinoma, 15 (27.2\%) had adenocarcinoma cell carcinoma, and 14 (25.5\%) had mixed or uncharacterized lung cancer (based on biopsy diagnosis). In the ITCT group, 33 cases were male and 9 were female, and members were aged between 19 and 85 years old, with a median age of $55 \pm 12$. In this group, $6(14.3 \%)$ patients had adenocarcinoma, 17 (40.5\%) had squamous carcinoma, and $18(42.8 \%)$ had mixed or uncharacterized lung cancer (based on biopsy diagnosis). The baseline characteristics of the patients were well balanced between the two groups (Table 1).

\section{Efficacy evaluation}

The median OS (censored observations; surviving patients still in follow-up) was 11.23 months in the UMIPIC group and 5.62 months in the ITCT group (Table 2). This represents a significant difference between the two groups (5.61 months longer) $(P<0.01)$ (curves [Kaplan-Meier] for both groups are depicted in Figure 2A). With a statistically significant difference, the 6-month survival rate was $76.36 \%$

Table 2 Comparison of mean and median survival times between the ultra-minimum incision personalized intratumoral chemoimmunotherapy (UMIPIC) and intratumoral chemotherapy (ITCT) groups

\begin{tabular}{lllll}
\hline Group & $\mathbf{N}$ & $\begin{array}{l}\text { Mean survival/ } \\
\text { month }\end{array}$ & $\begin{array}{l}\text { Median survival/ } \\
\text { month }\end{array}$ & $\boldsymbol{P}$ \\
\hline UMIPIC & 55 & 12.66 & 11.23 & 0.0023 \\
ITCT & 42 & 7.59 & 5.62 & \\
\hline
\end{tabular}



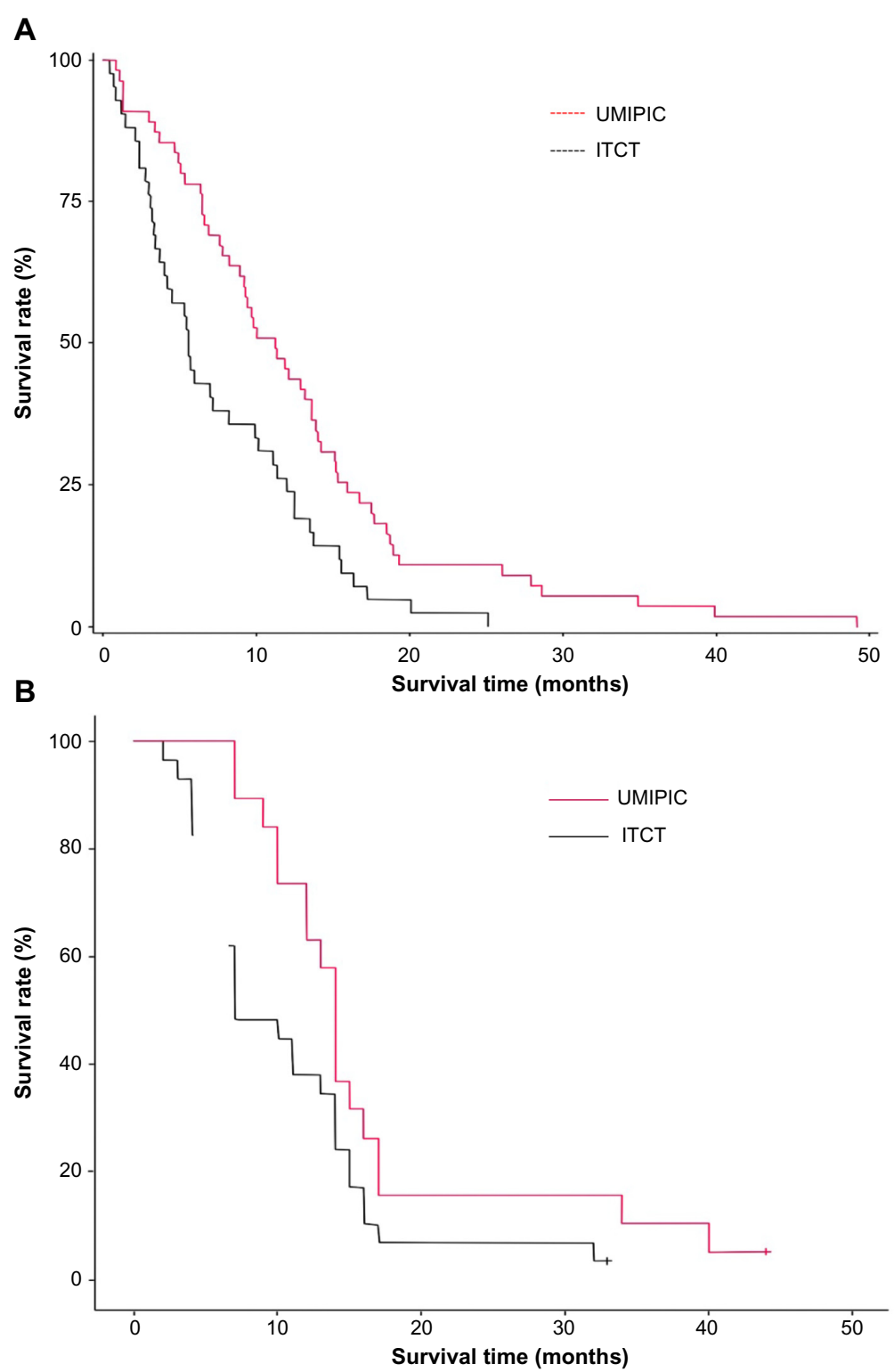

C

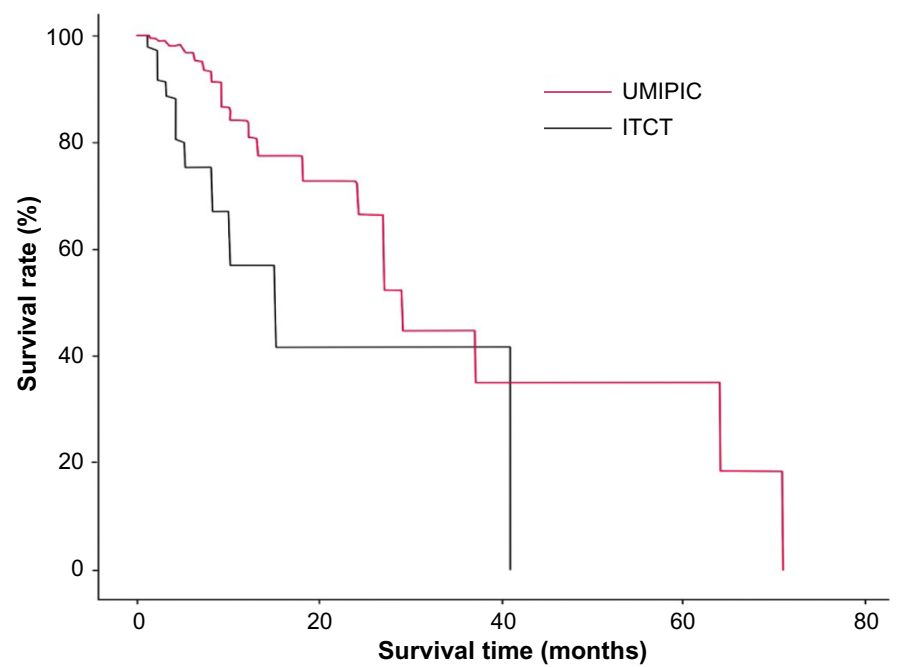

Figure 2 Ultra-minimum incision personalized intratumoral chemoimmunotherapy (UMIPIC) survival curve.

Notes: (A) Overall survival (OS) curves (Kaplan-Meier) and comparison of OS between the UMIPIC and intratumoral chemotherapy (ITCT) groups ( $P=0.0028)$. (B) Survival curves (Kaplan-Meier) of patients in the UMIPIC and ITCT groups who received two cycles of therapies. (C) Survival curves (Kaplan-Meier) of patients in the UMIPIC and ITCT who did not receive adjuvant treatment. 
(UMIPIC) versus $45.23 \%$ (ITCT) $(P<0.01)$ and 1 -year survival rate was $45.45 \%$ (UMIPIC) versus $23.81 \%$ (ITCT) $(P<0.05)$ (Table 3$)$.

Notably, members of the UMIPIC group who received two cycles of treatment had a survival advantage over those in the ITCT group who received two cycles of treatment. We evaluated the efficacy of two cycles of treatment in UMIPIC and ITCT refined groups, and this showed that the 6-month survival rate was $100 \%$ versus $72.41 \%(P<0.05)$ and the 1 -year survival rate was $73.68 \%$ versus $37.93 \%$, respectively $(P<0.05)$ (Tables 4 and 5 ). The median OS was 14 months for UMIPIC and 7 months for ITCT, representing a significant difference ( 7 months longer) between UMIPIC and ITCT patients who received two cycles of treatments $(P<0.01)$ (curves [Kaplan-Meier] for all groups are depicted in Figure 2B). Two cycles of treatment demonstrated more powerful debulking in the main tumor mass than a single cycle of treatment, and provided a better balance point for immunological power to eradicate more tumor cells (which are unaffected by UMIPIC) after UMIPIC killed the main mass of the tumor.

In the two groups, the complete survival data of 33 patients receiving UMIPIC $(\mathrm{N}=20)$ or ITCT $(\mathrm{N}=13)$ without adjuvant therapy (surgery, radiotherapy, or chemo-radiotherapy) were also analyzed, and a statistical difference in median OS of 11 months versus 4 months ( 7 months longer, $P<0.05$ ) (Table 6 ) between the UMIPIC and ITCT groups, respectively, was shown. The 6-month survival rate was $80 \%$ with UMIPIC versus $30.1 \%$ with ITCT $(P<0.05)$, and the 1 -year survival rate was 50\% with UMIPIC versus $15.38 \%$ with ITCT $(P<0.05$; Table 7 and curves depicted in Figure 2C). Comparisons of UMIPIC with ITCT (Tables 6 and 7) suggest that UMIPIC monotherapy has a better impact on patient survival rate than ITCT.

The response rates $(\mathrm{CR}+\mathrm{PR}+\mathrm{SD} /$ total $)$ were $81.8 \%$ and $83.3 \%$ in the UMIPIC and ITCT groups, respectively, indicating no significant difference $(P=0.055)$ (Table 8$)$. It is necessary to note that the slight size increase of the tumor mass observed clinically in both groups at first CT examination was likely due to inflammatory response

Table 3 Comparison of 6-month and I-year survival times between the ultra-minimum incision personalized intratumoral chemoimmunotherapy (UMIPIC) and intratumoral chemotherapy (ITCT) groups

\begin{tabular}{lllllllll}
\hline Group & $\mathbf{N}$ & \multicolumn{3}{c}{ 6-month survival rate } & & \multicolumn{3}{c}{ I-year survival rate } \\
\cline { 3 - 5 } \cline { 7 - 9 } & & $\%$ & $\chi^{2}$ & P-value & & $\%$ & $\chi^{2}$ & P-value \\
\hline UMIPIC & 55 & 76.36 & 9.885 & 0.0016 & & 45.45 & 4.838 & 0.028 \\
ITCT & 42 & 45.23 & & & & 23.81 & & \\
\hline
\end{tabular}

Table 4 Comparison of mean and median survival times with two cycles of therapies between the ultra-minimum incision personalized intratumoral chemoimmunotherapy (UMIPIC) and intratumoral chemotherapy (ITCT) groups

\begin{tabular}{|c|c|c|c|c|c|}
\hline \multirow[t]{2}{*}{ Group } & \multirow[t]{2}{*}{$\mathbf{N}$} & \multirow{2}{*}{$\begin{array}{l}\text { Mean survival } \\
\text { (months) }\end{array}$} & \multirow{2}{*}{$\begin{array}{l}\text { Median survival } \\
\text { (months) }\end{array}$} & \multicolumn{2}{|l|}{$t$-test } \\
\hline & & & & $\bar{T}$ & $P$-value \\
\hline UMIPIC & 19 & 16.79 & 14 & 2.806 & 0.007 \\
\hline ITCT & 29 & 10.66 & 7 & & \\
\hline
\end{tabular}

induced by coagulation, while after the addition of hapten in UMIPIC, more immunological response activities were induced on inflammations. ${ }^{10,11}$ Encouragingly, some of the remarkable responses in advanced cancer patients provided proof of the greatest effect on patients in the UMIPIC group (Figure $3 \mathrm{~A}$ and $\mathrm{B}$ ).

\section{Complications}

The related complications included temporary mild fever (not over $38^{\circ} \mathrm{C}$ ) for a few hours, minor pain at injection area, aerothorax in four cases, and leukocytopenia (within normal range) (Table 9). There were no patients with hemorrhage around the tumor or observed needle track after therapy. No significant systematic or local adverse effects were observed, and side effects such as myeloid suppression, neutropenia, thrombocytopenia, GI toxicity, and apparent loss of hair or appetite were not observed.

\section{Discussion}

Worldwide, lung cancer is still one of the major deadly diseases. Local treatment, like surgery and radiotherapy, is the primary curative therapy for patients in the early stages of lung cancer. Approximately $54 \%$ of patients present a metastasis at diagnosis due to lack of clinical symptoms at the early stages, which tends to result in an extremely poor prognosis with an overall 5 -year survival rate of $3.8 \% .^{12}$ For most advanced lung cancers, standard chemotherapy involving pemetrexed, ${ }^{13,14}$ oxaliplatin, ${ }^{15}$ and docetaxel, ${ }^{16}$ is generally the mainstream of management, but apparently this has reached a plateau with disappointing outcomes. ${ }^{17}$ Despite

Table 5 Comparison of 6-month and I-year survival times with two cycles of therapies between the ultra-minimum incision personalized intratumoral chemoimmunotherapy (UMIPIC) and intratumoral chemotherapy (ITCT) groups

\begin{tabular}{|c|c|c|c|c|c|c|c|}
\hline \multirow[t]{2}{*}{ Group } & \multirow[t]{2}{*}{$\mathbf{N}$} & \multicolumn{3}{|c|}{ 6-month survival rate } & \multicolumn{3}{|c|}{ I-year survival rate } \\
\hline & & $\%$ & $\chi^{2}$ & $P$-value & $\%$ & $\chi^{2}$ & $P$-value \\
\hline UMIPIC & 19 & 100.00 & 6.290 & 0.012 & 73.68 & 5.880 & 0.015 \\
\hline ITCT & 29 & $72.4 I$ & & & 37.93 & & \\
\hline
\end{tabular}


Table 6 Comparison of mean and median survival times without adjuvant treatments between the ultra-minimum incision personalized intratumoral chemoimmunotherapy (UMIPIC) and intratumoral chemotherapy (ITCT) groups

\begin{tabular}{llllll}
\hline Group & $\mathbf{N}$ & $\begin{array}{l}\text { Mean survival } \\
\text { (month) }\end{array}$ & $\begin{array}{l}\text { Median survival } \\
\text { (month) }\end{array}$ & $\mathbf{T}$ & P-value \\
\hline UMIPIC & 20 & 19.1 & II & - & - \\
ITCT & 13 & 7.54 & 4 & 2.177 & 0.037 \\
\hline
\end{tabular}

the introduction of a series of targeted drugs for patients with epidermal growth factor receptor mutations (gefitinib or erlotinib) ${ }^{18,19}$ and ALK rearrangement (crizotinib) ${ }^{20}$ in the past decade, the survival rate still has not been significantly improved. Today, immunotherapeutic interventions, including vaccine therapy derived from lung cancer cell lines (or tumor-associated antigens) and immune-stimulatory checkpoint antibodies, although traditionally not considered possible treatments for tumors, may improve outcomes in lung cancer. Moreover, the combination of immunotherapy and chemotherapy, or "chemoimmunotherapy", has been successfully applied clinically. ${ }^{21-23}$

"UMIPIC", described in this clinical study, is a patented therapeutic method for treating solid tumors, and was explored patients from this hospital with personalized dosages based on tumor size while utilizing patient-specific in vivo modified autologous tumor antigens as a self-vaccination to tumor-specific response. The regimen is a personalized and freshly prepared compound solution containing an oxidant, a cytotoxic drug, and hapten. Each component plays a vital role in the therapy.

"Intratumoral therapy", characterized as high local drug concentrations with minimal systematic toxicity, is an outstanding and attractive alternative to systematic treatment, with increasing evidence of its clinical benefits. ${ }^{24,25}$ The intratumoral delivery approach, integrated with the coagulation induced by the oxidant, can significantly increase the local accumulation of drugs (up to 10-100x that of systemic administration) ${ }^{8,26}$ Intratumoral therapy the oxidant acts as the main force in the debulking of the

Table 7 Comparison of 6-month and I-year survival times without adjuvant treatments between the ultra-minimum incision personalized intratumoral chemoimmunotherapy (UMIPIC) and intratumoral chemotherapy (ITCT) groups

\begin{tabular}{lllllllll}
\hline Group & $\mathbf{N}$ & \multicolumn{3}{c}{ 6-month survival rate } & & \multicolumn{3}{c}{ I-year survival rate } \\
\cline { 3 - 5 } & & $\%$ & $\chi^{2}$ & P-value & & $\%$ & $\chi^{2}$ & $P$-value \\
\hline UMIPIC & 20 & 80 & 4.5 I & 0.004 & & 50 & 4.08 & 0.043 \\
ITCT & 13 & 30.1 & & & & 15.38 & & \\
\hline
\end{tabular}

Table 8 Therapeutic response between the ultra-minimum incision personalized intratumoral chemoimmunotherapy (UMIPIC) and intratumoral chemotherapy (ITCT) groups

\begin{tabular}{|c|c|c|c|c|c|c|c|}
\hline \multirow[t]{2}{*}{ Effect } & \multicolumn{2}{|c|}{ UMIPIC } & \multicolumn{2}{|c|}{ ITCT } & \multicolumn{2}{|c|}{ Total } & \multirow[t]{2}{*}{$P$-value } \\
\hline & $\mathbf{N}$ & $\%$ & $\mathbf{N}$ & $\%$ & $\mathbf{N}$ & $\%$ & \\
\hline $\mathrm{CR}$ & 2 & 3.6 & 3 & 7.1 & 5 & 5.2 & 0.055 \\
\hline PR & 5 & 9.1 & 12 & 28.8 & 17 & 17.5 & \\
\hline SD & 38 & 69.1 & 20 & 47.6 & 58 & 59.8 & \\
\hline PD & 10 & 18.2 & 7 & 16.7 & 17 & 17.5 & \\
\hline Total & 55 & 100.00 & 42 & 100.00 & 97 & 100.00 & \\
\hline CR + PR (\%) & 12.7 & & 35.9 & & 22.7 & & \\
\hline $\begin{array}{l}\mathrm{CR}+\mathrm{PR}+ \\
\mathrm{SD}(\%)\end{array}$ & 81.8 & & 83.3 & & 82.5 & & \\
\hline
\end{tabular}

Abbreviations: $C R$, complete response; $\mathrm{PD}$, progressive disease; PR, partial response; SD, stable disease.

A

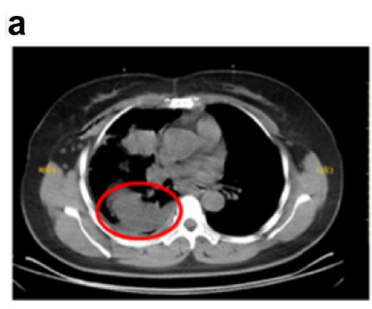

b

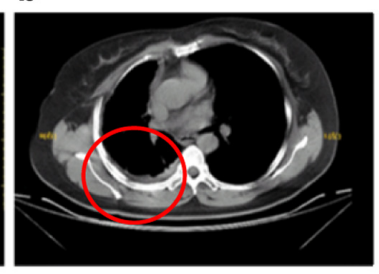

B

a

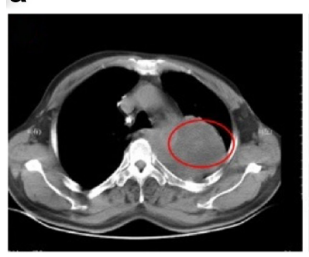

b

C

a

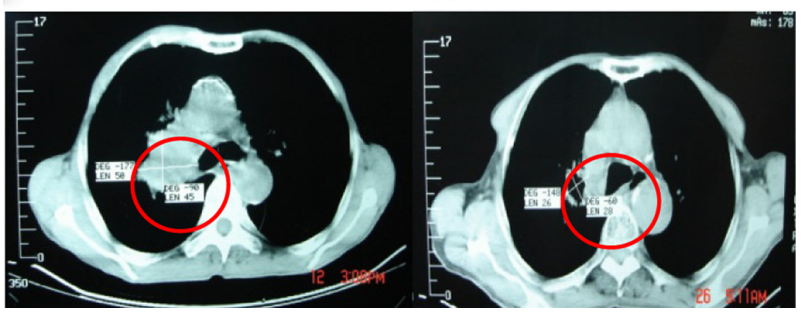

Figure 3 Clinical response of ultra-minimum incision personalized intratumoral chemoimmunotherapy (UMIPIC) in lung cancer.

Notes: (A) Response to UMIPIC therapy in lung tumor. The patient, a 49-year-old female, was diagnosed with lung cancer, adenocarcinoma, sited in the right lobe. She received a total of eight UMIPIC injections. (a) The tumor with a diameter of $64 \mathrm{~cm}$ pretreatment. (b) The cardinal of the tumor mass regressed to complete remission (CR) posttreatment. (B) Response to UMIPIC therapy in lung tumor. The patient, a 79-yearold male, had inoperable advanced lung cancer of squamous carcinoma at the time of diagnosis. He received a total of four UMIPIC treatments with adjuvant radiotherapy. (a) The tumor size was $6.7 \times 8.1 \mathrm{~cm}$ pretreatment. (b) The tumor regressed to partial remission posttreatment. (C) Response to UMIPIC therapy in central lung tumor. The patient, a 59-year-old female, was diagnosed with central lung cancer, adenocarcinoma, unresectable. She received a total of three UMIPIC injections. (a) The tumor with a diameter of $64 \mathrm{~cm}$ pretreatment. (b) The cardinal of the tumor mass regressed to $C R$ posttreatment, with a cavity of fibers at the primary site of the central lung cancer. The red circle denotes "the bulk of the tumor mass". 
Table 9 Related complications of the ultra-minimum incision personalized intratumoral chemoimmunotherapy (UMIPIC) and intratumoral chemotherapy (ITCT) groups

\begin{tabular}{llll}
\hline Complication & UMIPIC & ITCT & P-value \\
& N (\%) & N (\%) & \\
\hline Minimal local pain & $3(5.5)$ & $7(16.7)$ & 0.07 \\
Aerothorax & $2(3.6)$ & $2(4.7)$ & 0.78 \\
Leukocytopenia & $15(27.2)$ & $8(19.0)$ & 0.34 \\
Mild fever & I6 (29.1) & $7(16.7)$ & 0.15 \\
Slight cough & $6(10.9)$ & $7(16.7)$ & 0.41 \\
Nausea & I (I.8) & $2(4.7)$ & 0.40 \\
\hline
\end{tabular}

main tumor through coagulation, while the drug Ara-C continues to kill the residual tumors. The coagulation effect can effectively change the extracellular matrix and alter the morphological and biochemical components of the tumor, such as collagen, elastic fibers, reticular fibers, fibronectin, proteoglycans, hyaluronic acid, and other large molecules, creating a soft, semisolid, or solid mass with destroyed metabolism and induced fibrosis generation. It may also destroy the environmental conditions for tumor cell growth which was found in our previous animal experiment. ${ }^{27}$ More importantly, we not only found lymphocyte infiltration in the tumors, but also more positive cluster of differentiation (CD) $4^{+}$and $\mathrm{CD}^{+}$in the animals studied; and recently, we also found dendritic cells (DCs) and debris of tumor cells under electron microscopy (Figure 4). Therefore, coagulation is one of the major ways of improving drug utilization by extending the duration of drug action, as well as systematic drug exposure through sustained drug release, with greatly reduced toxicity ${ }^{28}$ and the induction of a possible immuno-capability against cancer cells in the body.

b

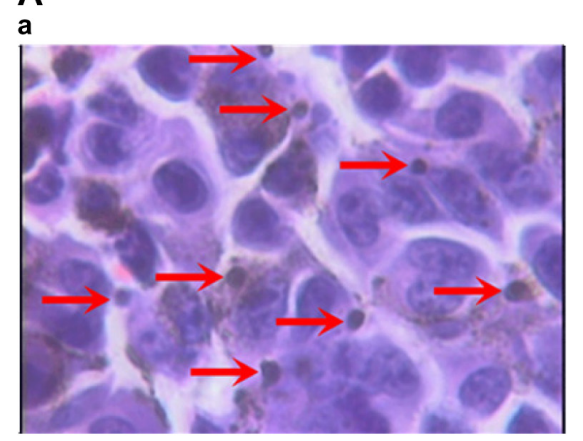

B

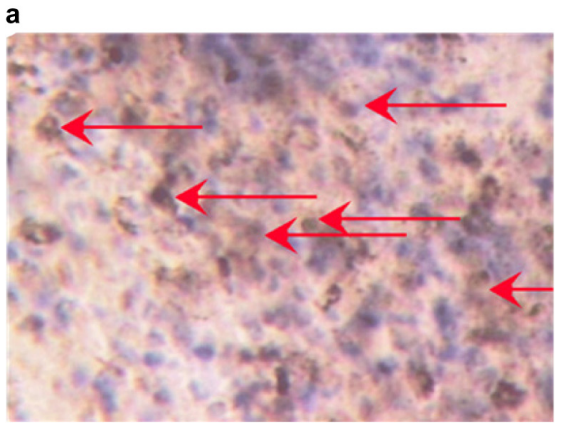

C

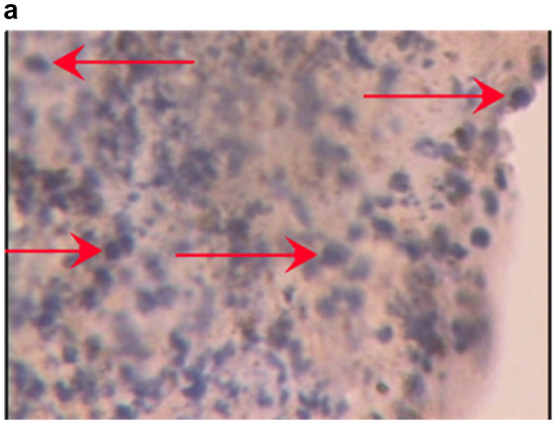

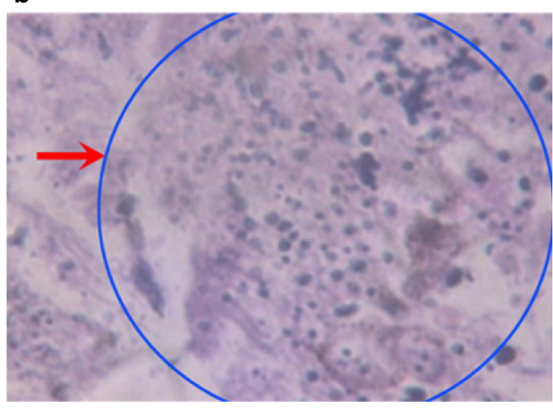

b

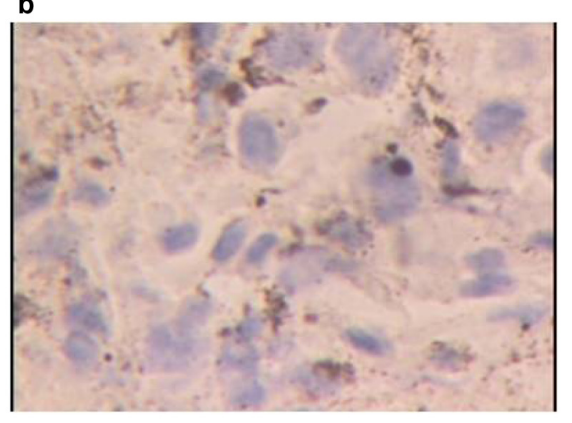

b

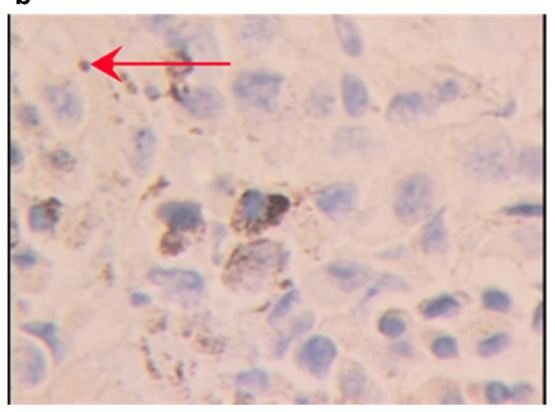

Figure 4 (Continued) 


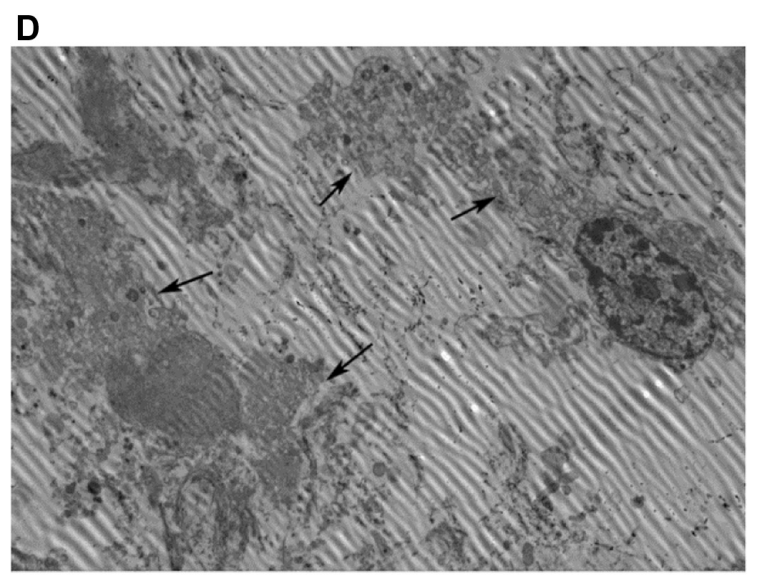

E

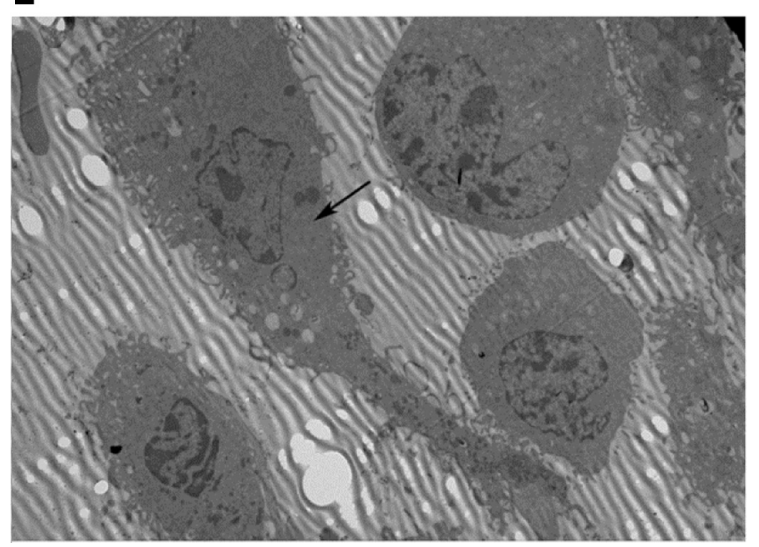

Figure 4 Localized inflammation with lymphocyte and dendritic cell (DC) infiltration in tumors.

Notes: (A) (a) With lymphocyte infiltration in the ultra-minimum incision personalized intratumoral chemoimmunotherapy (UMIPIC) survival group; (b) without lymphocyte infiltration in the intratumoral chemotherapy (ITCT) group. (B) (a) Cluster of differentiation (CD) $4^{+}$increase in tumors with UMIPIC treatment; (b) no CD4+ invasion in tumors with ITCT treatment. (C) (a) CD8 ${ }^{+}$increase in tumors with UMIPIC treatment; (b) no CD8 ${ }^{+}$invasion in tumors with ITCT treatment. (D) Tumor cell necrosis and debris under electron microscopy with UMIPIC treatment. (E) DCs under electron microscopy with UMIPIC treatment. The red arrows denote lymphocytes; the blue circle denotes aggregates of lymphocytes; and the black arrows denote dendritic cells.

A sustained release was observed in another clinical study. We separately performed intratumoral injection of 99Tcm-labeled Ara-C combined with an oxidant and 99Tcm Ara-C alone in two tumor masses in the same liver of a hepatocellular carcinoma patient. We found that drug retention was $82 \%$ versus $16 \%$ at 12 hours in each tumor and $60 \%$ versus $0 \%$ at 24 hours in each tumor after injection (Figure 5). Moreover, with the presence of the inflator, it is particularly important to note that the advantage of this approach, which includes high-sustaining and homogeneous drug diffusion in tumors, could present a satisfying clinical outcome. Compared with chemotherapy, the side effects
A

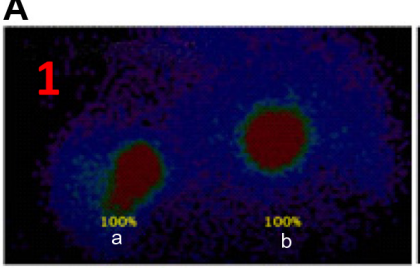

B

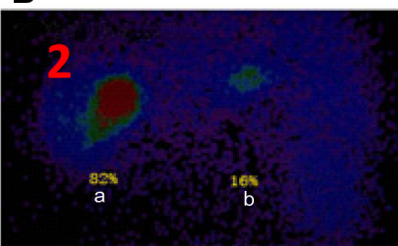

C

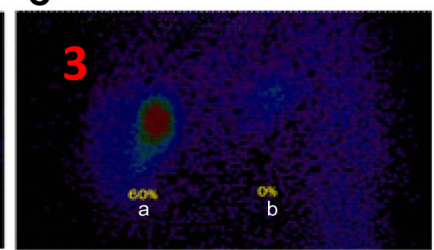

Figure 5 Comparison of retention rate of cytosine arabinoside (Ara-C) with and without intratumoral injection of oxidant.

Notes: (A) Fifteen minutes after the injection of Ara-C with cytotoxic oxidant (a group) and Ara-C alone (b group), with the retention rate of I00\% in both the (a) and (b) groups. (B) Four hours after injection of Ara-C with cytotoxic oxidant (a) and Ara-C alone (b), with retention rates of $82 \%$ and $16 \%$, respectively. (C) Twenty-four hours after injection of Ara-C with cytotoxic oxidant (a) and Ara-C alone (b), with the retention rates of $60 \%$ and $0 \%$, respectively. This was a clinical pharmacology study in a hepatocellular carcinoma patient with two tumor masses under nuclear camera; $99 \mathrm{~T} \mathrm{~cm}$-labeled Ara-C was successful, with a $99.9 \%$ labeling rate measured; $0.5 \mathrm{mCi}$ of $99 \mathrm{Tcm}$ Ara-C in cytotoxic oxidant (a) and the same dose of $99 \mathrm{Tcm}$ Ara-C in normal saline (b) were injected into two tumors in the liver and observed for $99 \mathrm{Tcm}$ isotope activities at different time points under single-photon emission computed tomography using a GEStarcom 400 . 
of UMIPIC include mild fever, local pain, and accidental aerothorax, which occurred in four cases in this study, but all with an improved quality of life. Aerothorax is one of the related complications of biopsy in the chest. ${ }^{29}$ Although it only happened in a few patients and these recovered spontaneously afterward. However, to reduce the possibility of aerothorax, it is suggested to keep the syringe filled with the compound solution before withdrawing the needle, and that patients be asked to inhale to block the needle track. The shortest distance and an ideal angle from which to puncture the tumor are the prerequisites to avoid complications. Latent metastatic cells brought along the needle track are killed by drops of compounded solution left in the needle track, so no cases with local metastasis were found in our study.

Creating an in situ vaccine depot in the tumor due to the release of tumor-specific antigens by the killed tumor cells is another intriguing factor in the process of ITCT. ${ }^{30}$ Furthermore, UMIPIC can not only induce the in vivo vaccine-like effect, but also enhance significantly the systematic immunity through the addition of hapten. When multiple autologous tumor antigens were released from the apoptotic or necrotic tumor cells induced by the coagulation and cytotoxic drug, cell death can be a priming event for T-cell response and can induce potent immunity. These cell deaths, called a "good death", 31,32 elicit a weak immune response as an in vivo selfvaccination promoted by immunologic modulator, that is, the small-molecule hapten inlaying the denatured tumor, and the modified cell debris or matrixes with tumor antigens became a new complex, more specific to the host immune system. This will generate stronger tumor antigens, referred to as an "autologous tumor vaccine", making the tumor itself more immunogenic to cancer cells.

In view of the optimistic survival advantage of UMIPIC therapy, we further analyzed the data for members of the UMIPIC group who also received conventional treatments. The median OS of patients in the UMIPIC group who received conventional treatments was 11.23 months (Table 2), and that of those who received UMIPIC alone was 11 months (Table 6), indicating the combination treatment may not sufficiently prolong the survival time compared with UMIPIC alone. In addition, according to clinical observation, the patients who received two cycles of treatment with UMIPIC had a median OS of 14 months (Table 4) and superior survival rate (Table 5) compared with those who received a single cycle of treatment. This may be attributed to the long-term immunological memory induced by the constitutive release of antigens, leading to a more effective antitumor response. With less tumor load, the debulking effect of UMIPIC resulted in better control of the residual cancer cells by immunological cells.

It is presumed that the inflammatory response, induced by coagulation and hapten, may also be involved with the antitumor immunity. The migration of APCs to the inflammatory tissue can enhance the capture and processing of tumor-associated antigens released from dead tumor cells to draining lymph nodes by APCs. This drives a desired antigen-specific immune response to further eradicate cancer cells at distant sites. ${ }^{33}$

\section{Conclusion}

The systemic immunity against patient-specific tumorassociated antigens was significantly boosted by increasing the presentation of antigens modified with hapten via APCs (including DCs and macrophage) to class I and class II pathways (to $\mathrm{CD}^{+}$and $\mathrm{CD}^{+} \mathrm{T}$-cells). Systemic immunity has the potential to generate immune effector responses and immune memory, ${ }^{34,35}$ and to recognize and destroy the residual lung cancer cells that initial coagulation missed in and around the primary tumor and micro-lesions after the UMIPIC. Examples of the elevation of systematic immune response were observed in our animal trial with high levels of $\mathrm{CD}^{+} / \mathrm{CD}^{+}{ }^{27}$

This clinical study showed that UMIPIC can induce more inflammatory responses in local tumors and showed a significantly prolonged survival time for patients with advanced lung cancer compared with ITCT in all aspects (Tables 2 to 7), and the addition of hapten in UMIPIC demonstrated a significant role as an immunological booster in terms of prolonged survival time.

In summary, UMIPIC for lung cancer is a noninvasive and potentially effective therapy with a satisfying profile of high specificity and prolonged survival time. It offers the prospect of tailoring treatments much more precisely and could lead to a better response, especially in patients with advanced-stage inoperable or drug-resistant types of lung cancer. More effective control of the disease is needed for us to investigate UMIPIC with two cytotoxic drugs and hapten under clinical study; it may reduce the number of injections and be more convenient to good practice.

\section{Disclosure}

The authors declare no conflicts of interest in this work; the authors have no outside funding source. 


\section{References}

1. Jemal A, Bray F, Center MM, Ferlay J, Ward E, Forman D. Global cancer statistics. Cancer J Clin. 2011;61(2):69-90.

2. American Cancer Society. Cancer Facts and Figures 2013. Atlanta, GA: American Cancer Society; 2013. Available from: http://www.cancer. org/research/cancerfactsstatistics/2013-cancer-facts-and-figures.pdf. Accessed October 8, 2014.

3. Mizuki N, Cryer SK, Okajima Y, et al. Tumoral cavitation in patients with non-small-cell lung cancer treated with antiangiogenic therapy using bevacizumab. Cancer Imaging. 2012;12:225-235.

4. Kim ES, Herbst RS, Wistuba II, et al. The BATTLE trial: personalizing therapy for lung cancer. Cancer Discov. 2011;1(1):44-53.

5. Nelson V, Ziehr J, Agulnik M, Johnson M. Afatinib: emerging nextgeneration tyrosine kinase inhibitor for NSCLC. Onco Targets Ther. 2013;6:135-143.

6. Ou SH, Bartlett CH, Mino-Kenudson M, Cui J, Iafrate AJ. Crizotinib for the treatment of ALK-rearranged non-small cell lung cancer: a success story to usher in the second decade of molecular targeted therapy in oncology. Oncologist. 2012;17(11):1351-1375.

7. Larsen JE, Cascone T, Gerber DE, Heymach JV, Minna JD. Targeted therapies for lung cancer: clinical experience and novel agents. Cancer J. 2011;17(6):512-527.

8. Yu B, inventor and assignee. Combinations and methods for treating neoplasms. United States patent US6811788 B2. November 2, 2004.

9. Duffaud F, Therasse P. [New guidelines to evaluate the response to treatment in solid tumors.] Bull Cancer. 2000;87(12):881-886. French.

10. Goldberg EP, Almond BA, Hadba AR, Cuevas BJ, York A, Enriquez I. Nano-mesosphere drug carriers for localized cancer chemotherapy. In: Technical Proceedings of the 2006 NSTI Nanotechnology Conference and Trade Show. Vol 2. Austin, TX: Nano Science and Technology Institute; 2006:1-4.

11. HogenEsch H. Mechanisms of stimulation of the immune response by aluminum adjuvants. Vaccine. 2002;20 Suppl 3:S34-S39.

12. Wright G, Manser RL, Byrnes G, Hart D, Campbell DA. Surgery for non-small cell lung cancer: systematic review and meta-analysis of randomised controlled trials. Thorax. 2006;61(7):597-603.

13. Paz-Ares L, de Marinis F, Dediu M, et al. Maintenance therapy with pemetrexed plus best supportive care versus placebo plus best supportive care after induction therapy with pemetrexed plus cisplatin for advanced non-squamous non-small-cell lung cancer (PARAMOUNT): a double-blind, phase 3, randomised controlled trial. Lancet Oncol. 2012;13(3):247-255

14. Baldwin CM, Perry CM. Pemetrexed: a review of its use in the management of advanced non-squamous non-small cell lung cancer. Drugs. 2009;69(16):2279-2302.

15. Cappuzzo F, Novello S, De Marinis F, et al. Phase II study of gemcitabine plus oxaliplatin as first-line chemotherapy for advanced non-small-cell lung cancer. Br J Cancer. 2005;93(1):29-34.

16. Komiyama K, Kobayashi K, Minezaki S, et al; Kanto Respiratory Disease Study Group. Phase I/II trial of a biweekly combination of S-1 plus docetaxel in patients with previously treated non-small cell lung cancer (KRSG-0601). Br J Cancer. 2012;107(9):1474-1480.

17. Schiller JH, Harrington D, Belani CP, et al; Eastern Cooperative Oncology Group. Comparison of four chemotherapy regimens for advanced non-small-cell lung cancer. N Engl J Med. 2002;346(2):92-98.
18. Mok TS, Wu YL, Thongprasert S, et al. Gefitinib or carboplatinpaclitaxel in pulmonary adenocarcinoma. NEngl J Med. 2009;361(10): 947-957.

19. Maemondo M, Inoue A, Kobayashi K, et al; North-East Japan Study Group. Gefitinib or chemotherapy for non-small-cell lung cancer with mutated EGFR. N Engl J Med. 2010;362(25):2380-2388.

20. Sasaki T, Jänne PA. New strategies for treatment of ALK-rearranged nonsmall cell lung cancers. Clin Cancer Res. 2011;11(23):7213-7218.

21. Reck M, Kaiser R, Mellemgaard A, et al. Nintedanib (BIBF 1120) plus docetaxel in NSCLC patients progressing after first-line chemotherapy: LUME Lung 1, a randomized, double-blind phase III trial. J Clin Oncol. 2013;31(Suppl):abstr LBA8011.

22. Sandler A, Gray R, Perry MC, et al. Paclitaxel-carboplatin alone or with bevacizumab for non-small-cell lung cancer. $N$ Engl J Med. 2006;355(24):2542-2550.

23. Reck M, von Pawel J, Zatloukal P, et al; BO17704 Study Group. Overall survival with cisplatin-gemcitabine and bevacizumab or placebo as first-line therapy for nonsquamous non-small-cell lung cancer: results from a randomised phase III trial (AVAiL). Ann Oncol 2010;21(9): 1804-1809.

24. Tohda Y, Iwanaga T, Takada M, et al. Intrapleural administration of cisplatin and etoposide to treat malignant pleural effusions in patients with non-small cell lung cancer. Chemotherapy. 1999;45(3):197-204.

25. Jackson JK, Gleave ME, Yago V, Beraldi E, Hunter WL, Burt HM. The suppression of human prostate tumor growth in mice by the intratumoral injection of a slow-release polymeric paste formulation of paclitaxel. Cancer Res. 2000;60(15):4146-4151.

26. Brincker H. Direct intratumoral chemotherapy. Crit Rev Oncol Hematol. 1993;15(2):91-98.

27. Qiong J, Baofa Y. Slow intra-tumor release of drugs on B16 melanoma in mice. J Shandong Univ. 2007;45(10):988-992.

28. Collins JM. Pharmacologic rationale for regional drug delivery. J Clin Oncol. 1984;2(5):498-504.

29. Zhenlu M, Guanghui C, Feng G, et al. CT guide percutaneous needle lung interstitial therapy in the treatment of advanced non-small cell lung cancer. Chin J Interv Imaging Ther. 2013;10:275-278.

30. Goldberg EP, Hadba AR, Almond BA, Marotta JS. Intratumoral cancer chemotherapy and immunotherapy: opportunities for nonsystemic preoperative drug delivery. J Pharm Pharmacol. 2002;54(2):159-180.

31. Nowak AK, Lake RA, Robinson BW. Combined chemoimmunotherapy of solid tumours: improving vaccines? Adv Drug Deliv Rev. 2006;58(8): 975-990.

32. Lake RA, Robinson BW. Immunotherapy and chemotherapy - a practical partnership. Nat Rev Cancer. 2005;5(5):397-405.

33. Ribas A, Butterfield LH, Glaspy JA, Economou JS. Current developments in cancer vaccines and cellular immunotherapy. J Clin Oncol. 2003;21(12):2415-2432.

34. Dredge K, Marriott BJ, Todryk SM, Dalgleish AG. Adjuvants and the promotion of Th1-type cytokines in tumour immunotherapy. Cancer Immunol Immunother. 2002;51(10):521-531.

35. Ho MY, Tang SJ, Sun KH, Yang W. Immunotherapy for lung cancers. J Biomed Biotechnol. 2011;2011:250860.

\section{Publish your work in this journal}

Lung Cancer: Targets and Therapy is an international, peer-reviewed, open access journal focusing on lung cancer research, identification of therapeutic targets and the optimal use of preventative and integrated treatment interventions to achieve improved outcomes, enhanced survival and quality of life for the cancer patient. Specific topics covered in the journal include: Epidemiology, detection and screening;
Cellular research and biomarkers; Identification of biotargets and agents with novel Submit your manuscript here: http://www.dovepress.com/lung-cancer-targets--therapy-journa
Dovepress

mechanisms of action; Optimal clinical use of existing anticancer agents, including combination therapies; Radiation and surgery; Palliative care; Patient adherence, quality of life, satisfaction; Health economic evaluations. The manuscript management system is completely online and includes a very quick and fair peer-review system. Visit http://www.dovepress.com/testimonials.php to read real quotes from system. Visit http:/
published authors. 